\title{
Box-Counting Method of 2D Neuronal Image: Method Modification and Quantitative Analysis Demonstrated on Images from the Monkey and Human Brain
}

\author{
Nemanja Rajković, ${ }^{1}$ Bojana Krstonošić, ${ }^{2}$ and Nebojša Miloševićc ${ }^{1}$ \\ ${ }^{1}$ Department of Biophysics, School of Medicine, University of Belgrade, Višegradska 26/2, 11000 Belgrade, Serbia \\ ${ }^{2}$ Department of Anatomy, School of Medicine, University of Novi Sad, Hajduk Veljkova 21, 21000 Novi Sad, Serbia \\ Correspondence should be addressed to Nebojša Milošević; mtn@med.bg.ac.rs
}

Received 24 January 2017; Revised 28 March 2017; Accepted 9 April 2017; Published 8 May 2017

Academic Editor: Dong Song

Copyright (C) 2017 Nemanja Rajković et al. This is an open access article distributed under the Creative Commons Attribution License, which permits unrestricted use, distribution, and reproduction in any medium, provided the original work is properly cited.

\begin{abstract}
This study calls attention to the difference between traditional box-counting method and its modification. The appropriate scaling factor, influence on image size and resolution, and image rotation, as well as different image presentation, are showed on the sample of asymmetrical neurons from the monkey dentate nucleus. The standard $\mathrm{BC}$ method and its modification were evaluated on the sample of 2D neuronal images from the human neostriatum. In addition, three box dimensions (which estimate the space-filling property, the shape, complexity, and the irregularity of dendritic tree) were used to evaluate differences in the morphology of type III aspiny neurons between two parts of the neostriatum.
\end{abstract}

\section{Introduction}

The fractal analysis, a modern mathematical method of measuring complexity in nature [1], is initially derived from fractal geometry [2]. The concepts of fractal geometry are being used in diverse research areas [3] and particularly are proven to be useful tool as quantitative methods for image analysis in medical science $[2,4]$. According to the fractal geometry, fractal analysis could be though, at present, as an experimental technique which calculates simple parameter: the fractal dimension (FD) of an object [5]. Previous work on fractal analysis represented traditionally a $2 \mathrm{D}$ analysis [5], focusing primarily on the object's border, structure, and indirectly object's function [2].

In fractal analysis, there are several different methods which calculate FD of object in a plane, but all of them can be summarized in two basic approaches: length-related and mass-related methods [3]. Using a different method has led to difficulties in comparing the results, because each method gives slightly different results when analyzing the same structure $[2,6]$. As traditional caliper methods are typically time-consuming, the need for other practicable techniques emerges [5]. One of them is traditional boxcounting method (BC method) which is based on concept of "covering" the image with rectangular coordinate grid [2]. Although this method is not suitable for measuring length, as well as other features of patterns, it is the best technique for estimating the FD. For that reason BC method is commonly used among other fractal techniques $[2,4,5]$.

Now, traditional BC method is suitable method for measuring FDs of real objects $[5,7]$. Thus, the first aim of this study was to modify standard BC method, using the appropriate scaling factor, image size, resolution, and rotation on 2D neuronal images from the monkey dentate nucleus. Having in mind modification procedure, this study could be seen as extension of previous investigations $[5,7]$, but here all conclusions were presented on asymmetrical neurons only. The second aim was to explore the differences in the morphology of the type III aspiny neurons from two parts 
of the human neostriatum, using standard and modified BC method.

\section{Materials and Methods}

Drawings of the Golgi-impregnated multipolar neurons of the monkey dentate nucleus were taken from the experimental data published in the book [8], in which is, also, described the histological procedure. The neuronal images were grouped according to Chan-Palay scheme of neuronal types [8]: 13 images of large "prickly" neurons, 18 images of boundary neurons, 24 images of asymmetrical neurons, and 22 images of "columnar" neurons [9].

Further, images from the neostriatum were collected from thirty human brains obtained from medicolegal forensic autopsies of adult bodies, free of neurological diseases [10]. The material was collected from 2008 to 2009 in the Center for Forensic Medicine, Toxicology and Molecular Genetics at the Clinical Center of Vojvodina (Serbia). As noted above, a total of 552 neurons (301 cells of the putamen and 251 cells of the caudate nucleus) were observed from both cerebral hemispheres [10]. From this population, 24 cells of the type III aspiny neurons [10] were selected for further analysis due to their unique dendritic arbor (low density of dendritic branching and giant dendritic domain [10]). The research was performed in accordance with the ethical standards defined by the 1964 Declaration of Helsinki, with the approval of the Ethics Committee of the University of Novi Sad (Faculty of Medicine, Serbia) [10].

2.1. Image Acquisition and Preprocessing. As we stated previously (Section 2), a total of 77 drawings from the monkey dentate nucleus were converted into digitized images using a scanner (Mustek 1200, Mustek System Inc., Taiwan) with a resolution of $600 \mathrm{dpi}$ [9]. During the scanning, the size of each image was rescaled to A5 format due to the restriction of fractal software (particularly, BC method in Image J). To investigate $\mathrm{BC}$ methodology, we standardized this sample following similar number of primary dendrites, area, length, and density of dendrites.

Furthermore, taking into consideration width and height of the neuronal images, from the total of 77 images, 14 images were selected and classified in the way that the type 1 neurons, which included eight asymmetrical neurons, had a greater width than the height, and the type 2 neurons, which included six asymmetrical neurons, had a greater height than the width. Their schematic representation is shown in Figures 1 (a) and 1(b).

In addition, 24 images of the type III aspiny neurons from the human neostriatum [10], 15 neuronal images from the putamen (Figure 1(c)), and 9 neuronal images from the caudate nucleus (Figure 1(d)), were analyzed with the standard and the modified BC method. After the histological sections of each obtained neuron were analyzed using a light microscope "Leica DC 100" (Leica Microsystems, Wetzlar, Germany) at a magnification of 40x, the images of neurons were transformed into digital images using the digital camera "Leica DC 100," with the software package "Digital Camera Systems" (Leica Microsystems, Heerbrugg, Switzerland)
$[10,11]$. Depending on the somal size and dendritic arborization, each neuron was recorded in 4-20 focal planes. The digital images of these focal planes were loaded into Image $J$ and using the "ZProject" command, the images were projected onto an image stack along the axis perpendicular to the image plane $[10,11]$.

Image processing was carried out using the public domain Image $J$ software (https://imagej.nih.gov/ij/). By using the corresponding tools of that software, the axons and spines were removed from digital images of neurons, and each dendrite was filled with pixels.

2.2. Standard BC Method. Segment-counting method [5], a type of fractal analysis methods, is at the same time robust and time-consuming [7]. For this reason, the need for more handsome methods emerges, and box-counting [3] appears to be the method which suitably measures fractal dimensions of real objects $[2,3,5,12]$. It is very similar (or sometimes, equal) to the idea from traditional calculus, when the "area of plane region" within any closed unformed boundaries should be measured [13], as the area is superimposed with a net of equivalent squares [14].

Traditional box-counting method "covers" the object with rectangular coordinate grid [3] and counts the number of boxes [2]. As each set of boxes is characterized by the square side $r$, the corresponding number of squares $(N)$ necessary to cover the pattern is presented as a function of $r$. Fractal dimension (i.e., box dimension) is determined as the slope of the log-log relationship between $N$ and $r$. Strictly mathematically "speaking," the lower and upper box dimensions of a subset $F \subset R^{n}$ are, respectively, defined by

$$
\begin{aligned}
& \underline{\operatorname{dim}}_{B}(F)=\varliminf_{\delta \rightarrow 0} \frac{\log N_{\delta}(F)}{-\log \delta}, \\
& \overline{\operatorname{dim}}_{B}(F)=\varlimsup_{\delta \rightarrow 0} \frac{\log N_{\delta}(F)}{-\log \delta},
\end{aligned}
$$

and if lower and upper values are equal, then the common value is referred to as the box-counting dimension of $F$ and is denoted by

$$
\operatorname{dim}_{B}(F)=\lim _{\delta \rightarrow 0} \frac{\log N_{\delta}(F)}{-\log \delta}
$$

where $N_{\delta}(F)$ can be the smallest number of cubes of side $\delta$ (naturally, in 3D) that covers $F$ or the largest number of disjoint cubes of side $\delta$ with centers in $F$ [15].

When $\mathrm{BC}$ method is applied on digitized images, it covers the image with a grid of square cells (with cell size $r$ ), where the cell size is expressed as the number of pixels (Figure 2(a)). The number of squares $N(r)$ needed to cover the image is given by a power law

$$
N(r)=\text { const } \cdot r^{-D_{B}},
$$

where $D_{B}$ is the box dimension ( $\mathrm{BD}$ in further text), obtained as an absolute value of the slope of the log-log relationship between $N(r)$ and $r$ [3] (Figure 2(b)). 


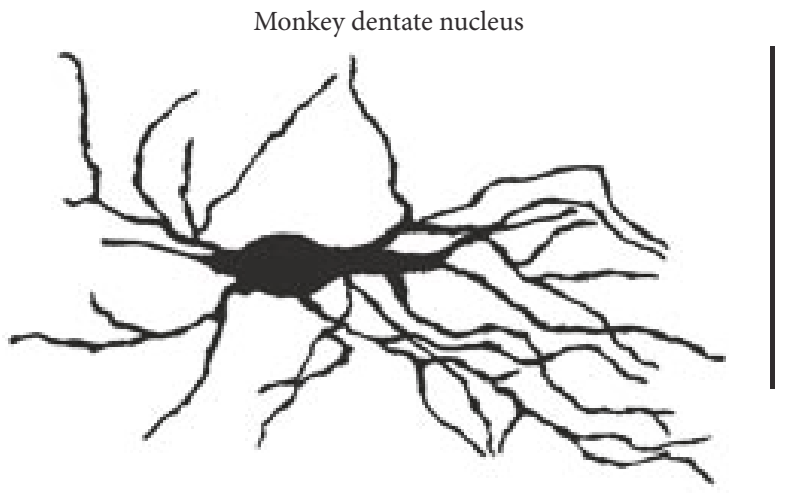

(a)

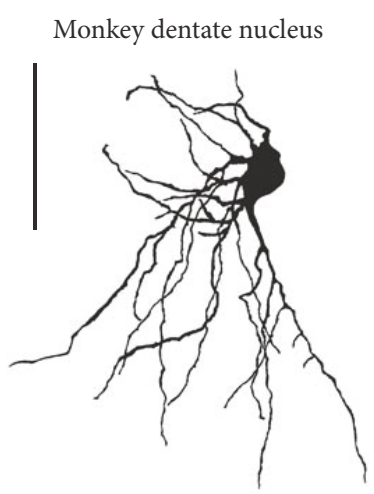

(b)

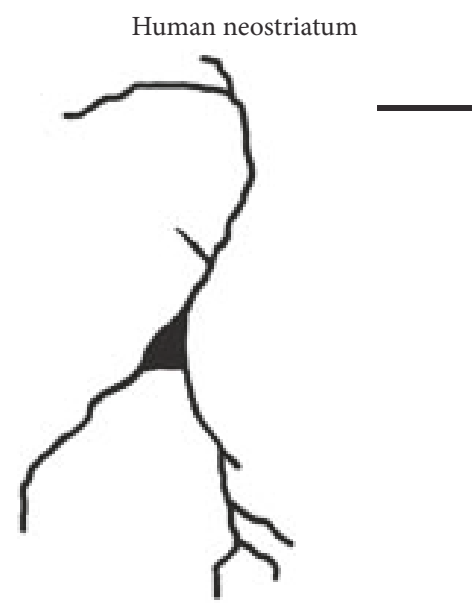

(c)

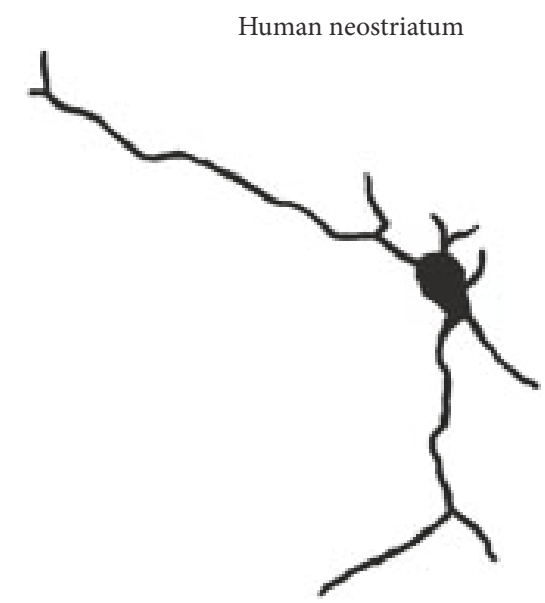

(d)

FIGURE 1: Representations of asymmetrical neurons from the monkey dentate nucleus: type 1 (a) and type 2 (b) cell. Original image can be found in Chan-Palay [8]. Representations of the type III aspiny neurons from the human neostriatum: image from the putamen (c) and caudate nucleus (d). All images are shown at 150 dpi with scale bar of $50 \mu \mathrm{m}$.

\subsection{The Image of the Neuron and BC Method}

2.3.1. Modification of Box Sizes. Even though mathematical fractal requires infinite orders of magnitude of the scaling [1], various structures in nature have a finite number of decades between a high and a low cut-off scale [2]. For instance, previous studies $[5,16]$ promote a hypothesis that the $2 \mathrm{D}$ neuronal images could be considered fractal over several decades of scale, if the box sizes are scaled as a power of 2. In addition, they present results of standard BC method, using arithmetical, geometrical, and random progression of box sizes $[5,16]$ where statistical evaluation of the correlation coefficient of fitted line has shown that it is different from zero with a very high significance $(p<0.0001)$. Despite very high value of correlation coefficient, choosing the size of boxes as a finite increasing geometric progression, compared to arithmetical progression, represents better solution of fitting problem, because in this case the starting object will fulfill all conditions of fractal analysis [5].
Finally, standard BC method should be modified as follows: the box sizes should be taken from $2^{0}$ to $2^{k}$ pixel, where $k$ is the value for which $N$ is equal to one (Figure 2(c)). In that case, besides different value of $D_{B}$, the relationship between $\log N$ and $\log r$ was linear on more than two decades of the range [2], when correlation coefficient of fitted line was statistically evaluated.

2.3.2. Influence of the Neuronal Image Size and Resolution. The fractal analysis of the same cells at different resolutions returned different FD values, even when all other parameters are kept constant [2]. What is more, in their previous work Jelinek et al. [17] have concluded that cells scanned at low resolution had higher values of FD than those obtained at high resolution. Previous study tested this hypothesis in one manner [7]: 14 images of asymmetrical neurons from the monkey dentate nucleus were printed on A4 paper (the size of each image was $13 \times 18 \mathrm{~cm}$ ) and image was scanned from resolution of $100 \mathrm{dpi}$ to $1100 \mathrm{dpi}$. The choice of final resolution 


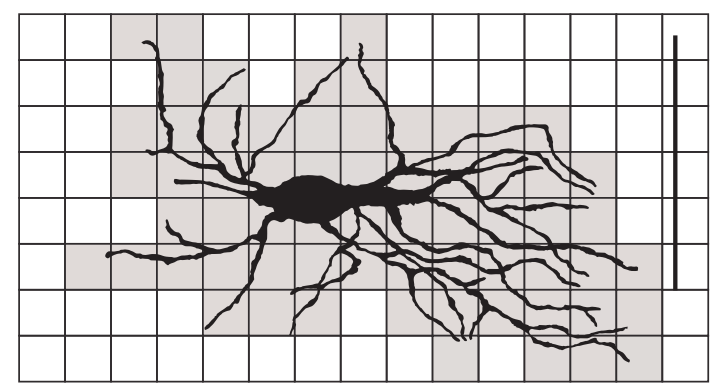

(a)

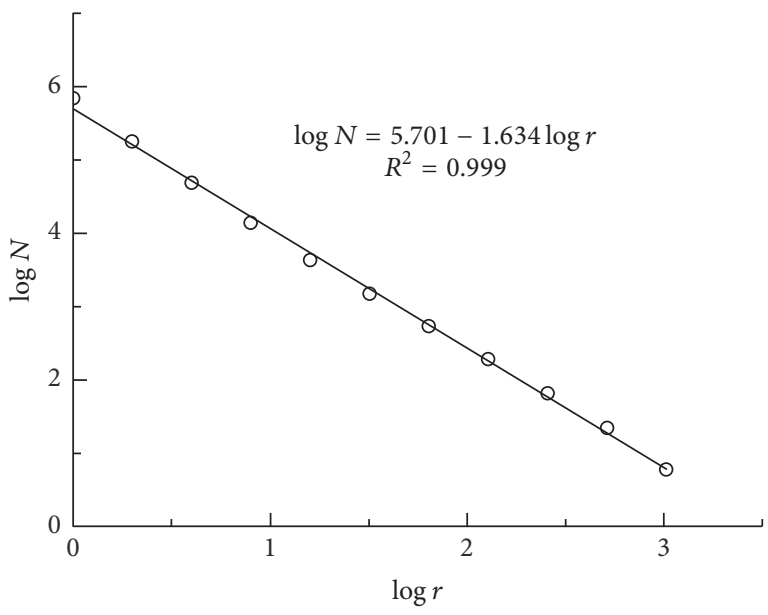

(b)

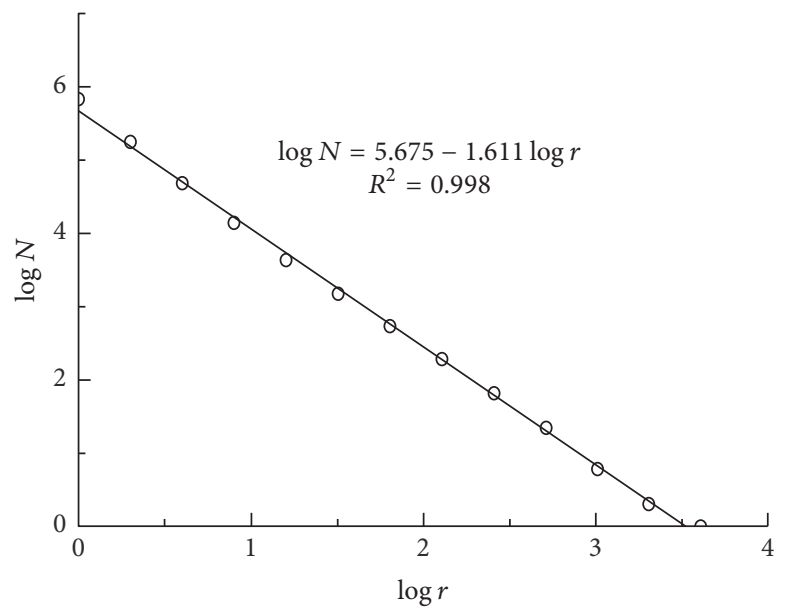

(c)

Figure 2: Application of the box-counting method to 2D image of type 1 neuron from the monkey dentate nucleus: (a) the whole image is covered with a set of squares and squares which cover dendrites are counted. (b) Log-log plot between numbers of squares $(N)$ and square size $(r)$ is fitted by a straight line. The square sizes $(r)$ are taken by geometric progression. (c) Modification of square sizes (from 1 to 4096 pixels) illustrates interval of scaling and accurate BD. The linear equation between $\log N$ and $\log r$ is shown in upper part of graphs, where $D_{B}$ is the absolute value of the slope and $R$ is the corresponding correlation coefficient.

was restricted by type of a scanner (Mustek 1200, Mustek Systems Inc., Taiwan). Results of this study suggested that mean $D_{B}$ increased with resolution, with extremely low value of the slope; therefore the resolution does not influence $B D$ value of the image [7].

Present study shows another step in investigating influence of image resolution (and, thus, different image size) on value of $D_{B}$. The same sample of images were initially scanned at $600 \mathrm{dpi}$, and for each image, resolution was digitally changed (increased or decreased). Thus, we gained two samples of asymmetrical neurons (type 1 and type 2) with the same interval of resolution as previous study (100 dpi-1100 dpi). Results confirmed conclusions presented in [7]: there was an increase in $D_{B}$ with the resolution, but again, with very low value of the slope $\left(10^{-4}\right.$ for type 1 and 0.9 $\times 10^{-4}$ for type 2). For both types of asymmetrical neurons results suggested that high resolution would maximize the resemblance between the digital and original drawings [7].
2.3.3. Image Rotation. The picture exhibits rotational symmetry if rotation by a specific angle around some central axis point can return the picture to its original configuration [18], and it is reasonable to imagine that the $D_{B}$ s of such picture in these positions have the same value. As for neuronal image, some findings reported unexpected results for images of neurons which possess lack in strong radial symmetry, that is, nonstellate neurons [18] or, that is, asymmetrical neurons. Previous study proposed possible explanation on how to perform exact calculation of $D_{B}$ [7]: first, the axis of rotation was created connecting two distant points of dendritic field area around the neuron [19]. Then, all images were continuously rotated from $0^{\circ}$ to $360^{\circ}$, increasing the angle by $15^{\circ} . D_{B}$ either increases or decreases and maximal (or minimal) $D_{B}$ was noticed in $45^{\circ}, 135^{\circ}, 225^{\circ}$, and $315^{\circ}$. The final $D_{B}$ was calculated as the mean of these four values.

Thus, to calculate accurate $D_{B}$ for asymmetrical neuronal images, we propose another modification of the $\mathrm{BC}$ method: 


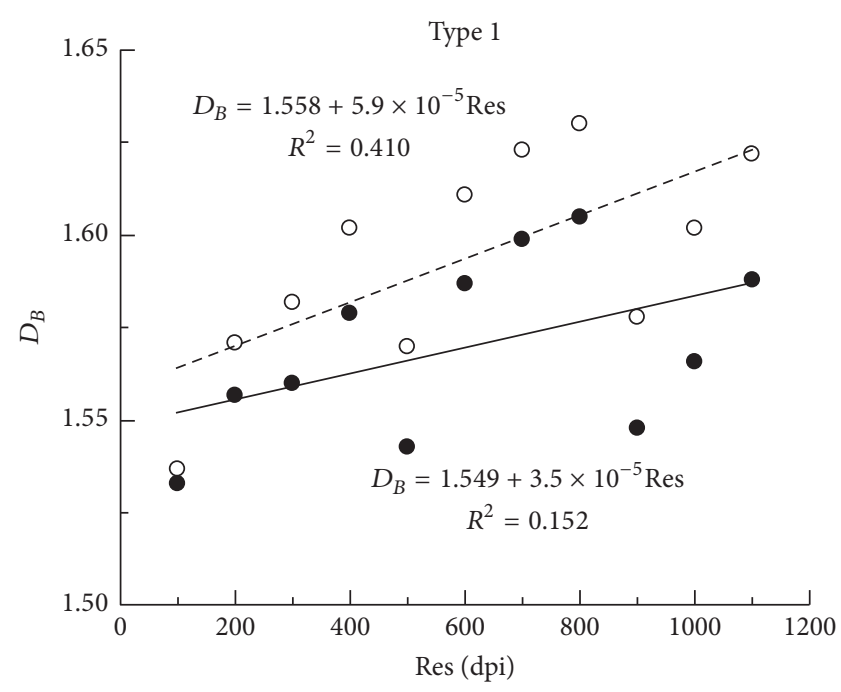

(a)

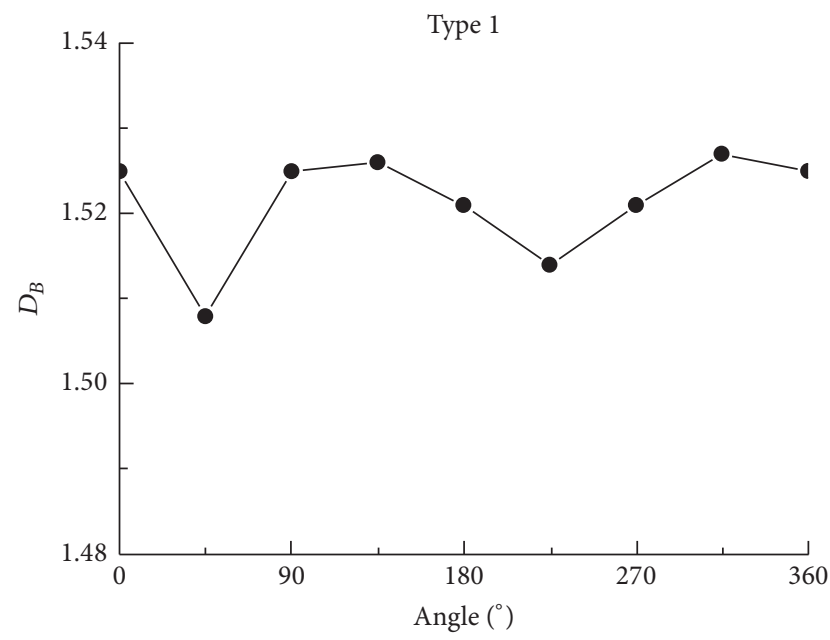

(c)

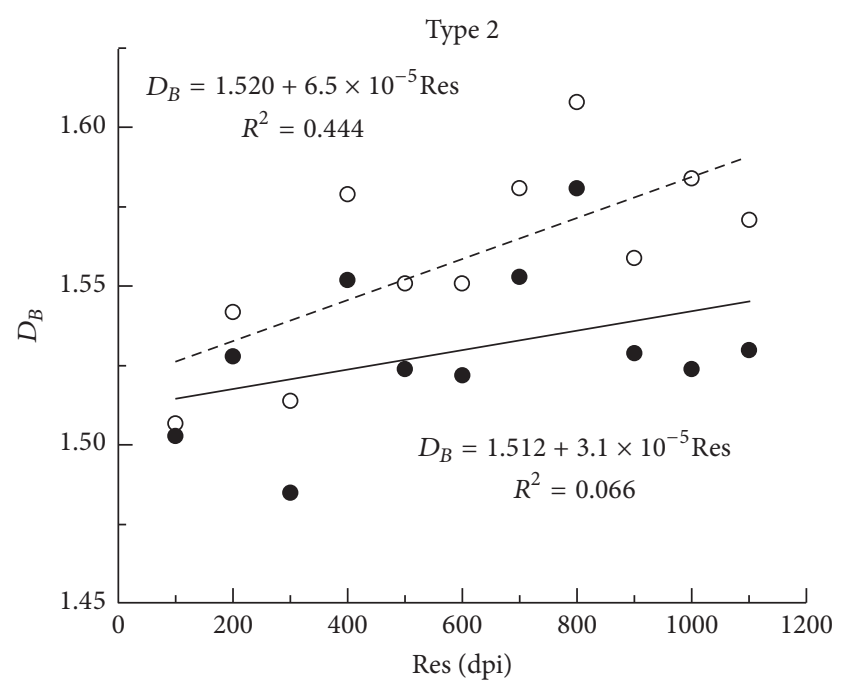

(b)

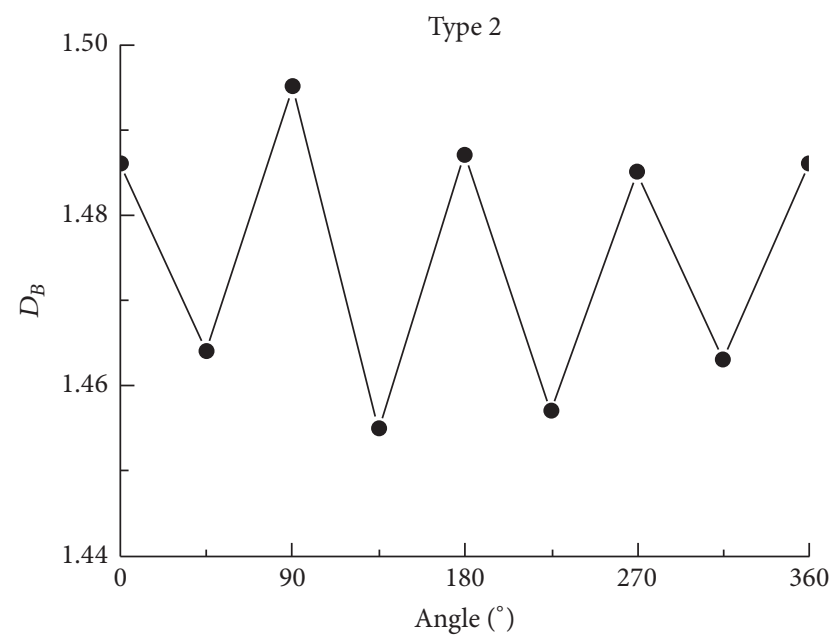

(d)

Figure 3: (a, b) Plots of $\left(D_{B}\right)_{\text {bin }}$ versus resolution for type 1 (a) and type 2 (b) neurons shown in Figure 1: full line is for scanned images and dashed line is for digital changes. The equations between $D_{B}$ and resolution, as well as correlation coefficients are inscribed in each graph. (c, d) Changes in $\left(D_{B}\right)_{\text {bin }}$ with an angle of rotation $(\alpha)$ for type $1(\mathrm{c})$ and type $2(\mathrm{~d})$ asymmetrical neurons of monkey dentate nucleus.

each image should be analyzed for symmetry. The axis of rotation should be constructed and the image should be rotated by four angles $\left(45^{\circ}+k \pi / 2\right.$, where $k=0,1,2$, and 3). In each position, apparent $D_{B}$ should be recorded and final (or precise) $D_{B}$ will be the mean of these values. Figures $3(c)$ and $3(\mathrm{~d})$ illustrate this procedure for type 1 and type 2 asymmetrical neurons from the monkey dentate nucleus.

2.3.4. Image Types and Corresponding BDs. When 2D RGB or grayscale image of the neuron is quantified by BC method, two characteristic image presentations are recognized: binary and outline (previously known as "silhouette" [3]) image. In any software for image analysis, the binary image represents compression of an initial image to two values (i.e., black and white values). Consequently, the outline image was created when one-pixel wide outline of foreground objects in a binary image was generated.
Previous study provides explanation on how $D_{B}$ can estimate an object's projection in plane [5]. Theoretically, when size of squares was reduced by two, the number of boxes multiplies previous number by 4 , and $D_{B}$ was equal to 2 with coefficient of determination equal to one. Thus, for $2 \mathrm{D}$ neuronal image, the value of $D_{B}$ estimates the area of its projection [5], but precisely it can be stated that $D_{B}$ estimates space-filling property of the neuron. Exactly, $D_{B}$ estimates how neuronal projection fills the plane defined by image size. Then again, the border of binary image (i.e., outline image), evaluates the irregularity in the shape of the image or precisely the value of $D_{B}$ shows how this value deviates from values of classic geometric figures or more complex forms.

There is another type of image, commonly used in earlier phase of digital image analysis, when images of neurons are drawn by camera lucida throughout dendritic axis [14]. It is known as skeleton image of the neuron [14], since they 


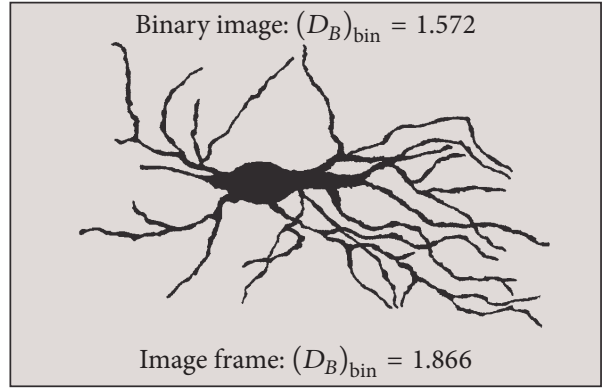

(a)

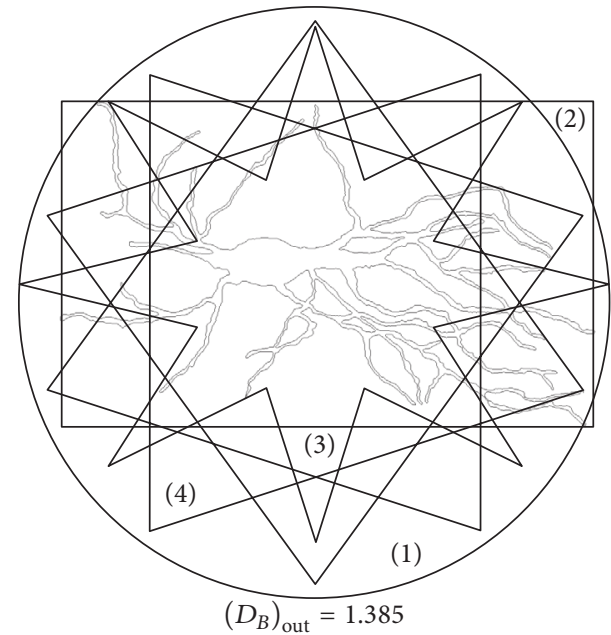

(1) $\left(D_{B}\right)_{\text {out }}=1.032 \quad$ (3) $\left(D_{B}\right)_{\text {out }}=1.135$

(2) $\left(D_{B}\right)_{\text {out }}=1.100$

(4) $\left(D_{B}\right)_{\text {out }}=1.210$

(b)

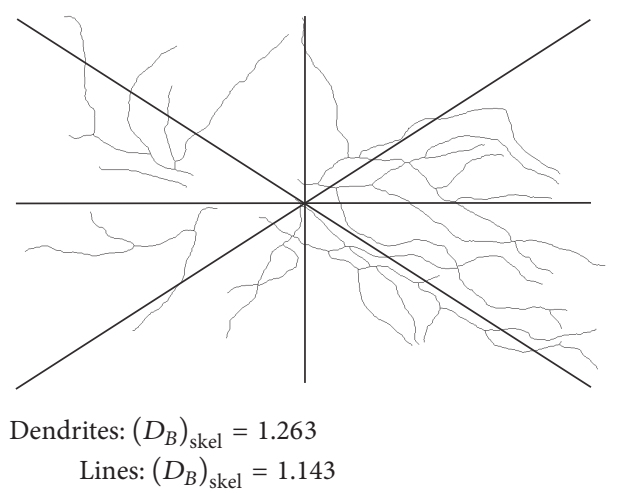

(c)

Figure 4: The binary (a), outline (b), and skeleton (c) images of asymmetrical neuron shown in Figure 1(a). For each image corresponding BD is presented along with theoretical values from which calculated BD has been compared, in order to evaluate three morphological properties of the neuron.

represent only the dendritic branching and do not reflect the other characteristic of complexity or border roughening $[2,20]$. In most software, command skeletonize works only with binary 2D images, removing pixels from the edges of objects until they are reduced to single-pixel-wide shapes.

Traditional BC method was modified when 2D neuronal images have been analyzed by using skeleton process [5], particularly when dendritic branching of neurons with thick dendrites and large cell bodies have been investigated [18]. The cell body on the binary image of neuron was digitally removed and remaining dendrites were sublimated in single pixel line while remaining artifacts were deleted $[2,5,14]$. The analysis of such image or $D_{B}$ of this kind of neuronal image precisely estimates both, dendritic branching pattern and dendritic aberration [5]. Figure 4 illustrates three BDs, which evaluate the space-filling property, shape, dendritic aberration, and complexity of dendritic tree for type 1 asymmetrical neurons from the monkey dentate nucleus.

\section{Results}

The morphology of 24 images from the human neostriatum (i.e., type III neurons) was analyzed by BC method in order to explore (i) the difference in BDs obtained by standard and modified BC method and (ii) the possible difference in BDs between two parts of the human neostriatum (putamen and caudate nucleus). BC method was done using Image J, after each image was saved in three different formats: binary (Process: Binary $\rightarrow$ Make binary), outline (Process: Binary $\rightarrow$ Outline), and skeleton (Process: Binary $\rightarrow$ Skeletonize) image and corresponding BDs were calculated. The skeleton process in Image J uses a thinning algorithm, thoroughly explained in the study of Zhang and Suen [21].

Analysis of the calculated $D_{B}$ s depends on whether the distribution is normal or not [22]. Regardless of the fact that the type III neurons were selected from normally distributed population [10], the number of neurons was relatively small 
TABLE 1: Box-counting analysis of 24 images of the type III aspiny neurons from the adult human neostriatum. The values of skewness $\left(a_{3}\right)$ and excess $(e)$ for binary $\left(\left(D_{B}\right)_{\text {bin }}\right)$, outline $\left(\left(D_{B}\right)_{\text {out }}\right)$, and skeleton $\left(\left(D_{B}\right)_{\text {skel }}\right)$ box dimensions, calculated by standard and modified BC method. $t_{c}$ is calculated $t$-value and $p$ is the significance level.

\begin{tabular}{|c|c|c|c|c|c|c|}
\hline & & $a_{3}{ }^{*}$ & $e^{*}$ & Mean \pm SE & $t_{c}{ }^{*}$ & $p$ \\
\hline \multirow{2}{*}{$\left(D_{B}\right)_{\text {bin }}$} & Standard & -0.292 & -1.352 & $1.43 \pm 0.01$ & \multirow{2}{*}{4.074} & \multirow{2}{*}{$<0.05$} \\
\hline & Modified & 0.401 & -1.930 & $1.393 \pm 0.008$ & & \\
\hline \multirow{2}{*}{$\left(D_{B}\right)_{\text {out }}$} & Standard & -0.335 & -1.094 & $1.186 \pm 0.009$ & \multirow{2}{*}{0.740} & \multirow{2}{*}{$>0.05$} \\
\hline & Modified & 0.550 & -0.382 & $1.194 \pm 0.007$ & & \\
\hline \multirow{2}{*}{$\left(D_{B}\right)_{\text {skel }}$} & Standard & 0.369 & -0.821 & $1.086 \pm 0.008$ & \multirow{2}{*}{0.359} & \\
\hline & Modified & 0.452 & -0.992 & $1.082 \pm 0.008$ & & \\
\hline
\end{tabular}

${ }^{*} \sigma_{3}=0.452, \sigma_{4}=0.768, t_{0.05}=2.069, t_{0.01}=2.807$, and $t_{0.001}=3.767$.

TABLE 2: Three box dimensions $\left(\left(D_{B}\right)_{\text {bin }},\left(D_{B}\right)_{\text {out }}\right.$, and $\left.\left(D_{B}\right)_{\text {skel }}\right)$ of the type III neuronal images from two parts of the human neostriatum. $t_{c}$ is calculated $t$-value and $t_{t}$ is tabulated $t$-value on $p=0.05$.

\begin{tabular}{|c|c|c|c|c|}
\hline \multirow{2}{*}{ Box dimension } & \multicolumn{2}{|c|}{ Type III neurons } & \multirow{2}{*}{$t_{c}$} & \multirow{2}{*}{$t_{t}$} \\
\hline & Caudate nucleus & Nucleus putamen & & \\
\hline$\left(D_{B}\right)_{\text {bin }}$ & $1.40 \pm 0.02$ & $1.387 \pm 0.008$ & 1.092 & \\
\hline$\left(D_{B}\right)_{\text {out }}$ & $1.19 \pm 0.01$ & $1.20 \pm 0.01$ & 0.379 & 2.074 \\
\hline$\left(D_{B}\right)_{\text {skel }}$ & $1.07 \pm 0.01$ & $1.09 \pm 0.01$ & 0.395 & \\
\hline
\end{tabular}

and character of the distribution could be tested with two statistical parameters: skewness $\left(a_{3}\right)$ and excess of distribution (e) [5]. In brief, the intervals of distributions are estimated when $a_{3}$ and $e$ were divided by the corresponding mean square errors $\left(\sigma_{3}\right.$ and $\left.\sigma_{4}\right)$. If the absolute value of the quotients $\sigma_{3} / a_{3}$ and $\sigma_{4} / e$ is less than or equal to 2 , then the data distribution can be considered as normal [5].

Table 1 shows values of $a_{3}$ and $e$ for three different $D_{B} s$ calculated with standard and modified box-count method. As the absolute ratios $\left(\sigma_{3} / a_{3}\right.$ and $\left.\sigma_{4} / e\right)$ are smaller than the critical value of 2 in all cases, thus, the calculated $D_{B} s$ can be expressed by the mean values and standard errors. As can be seen, the mean $\left(D_{B}\right)_{\text {bin }}$ and $\left(D_{B}\right)_{\text {skel }}$ were higher by standard $\mathrm{BC}$ method than the modified, while opposite conclusion can be drawn for mean $\left(D_{B}\right)_{\text {out }}$. Moreover, the difference between two methods was $0.4 \%$ for $\left(D_{B}\right)_{\text {skel }}, 0.7 \%$ for $\left(D_{B}\right)_{\text {out }}$, and $2.7 \%$ for $\left(D_{B}\right)_{\text {bin }}$. Further, only for $\left(D_{B}\right)_{\text {bin }}$ the difference was statistically significant.

The second task of this study was done by calculating three BDs using modified BC method. Table 2 shows means and standard errors for $\left(D_{B}\right)_{\text {bin }},\left(D_{B}\right)_{\text {out }}$, and $\left(D_{B}\right)_{\text {skel }}$ for the neuronal images of the putamen and the caudate nucleus. It looks that the images of neurons in the caudate nucleus have larger value of mean $\left(D_{B}\right)_{\text {bin }}$ than the images in the putamen, but for all three DBs no significant differences were found (Table 2).

\section{Discussion}

The extension of the concepts of fractal geometry toward the biomedical sciences has led to significant progress in understanding complex functional properties and structural features [23-29]. Once fractal geometry was formulated, many neuroscientists adopted fractal analysis as an appropriate method for objective quantitative analysis of neuronal structures [2, 30]. One of the advantages of using fractal analysis is its capacity to make a difference among neurons that differ in the complexity of their dendritic and axonal branching patterns $[2,3,7]$.

4.1. Modified BC Method. As Section 2.2 explains mathematical background of standard BC method, this paper presents its modification, particularly when $2 \mathrm{D}$ image of neuronal projection has been quantified (Section 2.3). Standard BC method was improved with the appropriate scaling factor (Section 2.3.1) and the image size and resolution have been explored (Section 2.3.2). Along with previous, this paper improves conclusion presented in the study of Ristanovic and coauthors [18], regarding image rotation and correct $D_{B}$ calculation (Section 2.3.3). The influence of image size, resolution, and rotation has been presented on images of asymmetrical cells from the monkey dentate nucleus.

The Methods section in this text shows significance of $D_{B}$ calculated for different presentations of the same neuronal image. Precisely, $D_{B}$ evaluates the space-filling property, perimeter of the neuron shape, and irregularity of dendrites.

In previous study [31], images from several mammalian spinal cords were investigated, with proposed scaling factor, and results undoubtedly showed that images from rat's and cat's spinal cords should be investigated, mainly, as binary images [31]. Another study summed up investigation of 76 images from the adult human dentate nucleus [5] with three types of BDs. $\left(D_{B}\right)_{\text {bin }}$ was subjected to the size of the dendritic field $\left(A_{\mathrm{DF}}\right)$ and $\left(D_{B}\right)_{\text {out }}$ was subjected to the circularity ratio $(M)$. The results demonstrated the fact that $\mathrm{BDs}$ are more sensitive than the size of dendritic field and circularity ratio $[5]$.

4.2. Aspiny Neurons of the Human Neostriatum. To illustrate proposed modification of the $\mathrm{BC}$ method, we choose images of aspiny neurons from the human neostriatum, precisely images of the type III neurons. Our pool of cells represents statistically small sample (less than 30 ), but these could be 
expected as previous study [10] postulates that this type of cells consists up to $10 \%$ of the whole population. However, this sample was enough to demonstrate the difference between standard and modified BC method. The difference was obvious (i.e., statistically significant) when binary images have been compared (Table 1).

Another task of this study was to investigate morphological differences of aspiny neurons between two parts of the neostriatum. Previous study [10] reported differences between neurons of the putamen and the caudate nucleus when dendritic field area and density of the dendritic trees were analyzed. This paper analyzes space-filling property, shape of the neuron, complexity, and irregularity of dendrites with modified BC method. Results look unequivocal: the binary $\mathrm{BD}$ is higher for the neuronal images of the caudate nucleus (while outline and skeleton BDs are lower) than the images of the putamen (Table 2), but without statistical significance. Such information leads us to the conclusion: either this sample of neurons was too small or the type III aspiny neurons from two parts of human neostriatum have similar morphology (i.e., the space-filling property and shape of the neuron, as well as dendritic complexity and aberration). However, we believe that further study with the large number of the type III neurons would provide more precise conclusions.

\section{Conclusion}

The main aim of the fractal analysis is to calculate the FD of an object and to ascertain the significance of the obtained value in terms of the complexity of the object. As for 2D image of the neuron, FD should quantify its morphology on the basis of how image is presented. However, the present study shows importance of image preprocessing, particularly when FD is calculated by $\mathrm{BC}$ method. BC modifications could be very important having in mind that $\mathrm{BC}$ method is, today, the most common procedure for calculation of FD.

\section{Conflicts of Interest}

The authors declare that there are no conflicts of interest regarding the publication of this paper.

\section{Acknowledgments}

This work was supported by the Ministry of Education and Science Republic of Serbia, Contracts no. III 41031. Assistance with all question/problems/ideas and solutions concerning fractal theory, fractal geometry, fractal analysis, and box-counting method by Professor Dušan Ristanović (Department of Biophysics, Medical school, University of Belgrade) is gratefully acknowledged.

\section{References}

[1] B. B. Mandelbrot, The Fractal Geometry of Nature, W.H. Freeman, New York, NY, USA, 1982.

[2] E. Fernández and H. F. Jelinek, "Use of fractal theory in neuroscience: methods, advantages, and potential problems," Methods, vol. 24, no. 4, pp. 309-321, 2001.
[3] T. G. Smith Jr., G. D. Lange, and W. B. Marks, "Fractal methods and results in cellular morphology - dimensions, lacunarity and multifractals," Journal of Neuroscience Methods, vol. 69, no. 2, pp. 123-136, 1996.

[4] A. Di Ieva, F. J. Esteban, F. Grizzi, W. Klonowski, and M. MartínLandrove, "Fractals in the neurosciences, part II: clinical applications and future perspectives," Neuroscientist, vol. 21, no. 1, pp. 30-43, 2015.

[5] N. T. Milošević, "Fractal analysis of two dimensional images: parameters of the space-filling and shape," in Proceedings of the 20th International Conference on Control Systems and Computer Science, Vol. 2: IAFA: Fractal Analysis of Medical Images. Los Alamitos: The Institute of Electrical and Electronics Engineers, I. Dumitrache, A. Magda Florea, F. Pop, and A. Dumitrascu, Eds., pp. 539-544, 2015.

[6] F. Caserta, W. D. Eldred, E. Fernandez et al., "Determination of fractal dimension of physiologically characterized neurons in two and three dimensions," Journal of Neuroscience Methods, vol. 56, no. 2, pp. 133-144, 1995.

[7] N. T. Milošević, "The morphology of brain neurons: boxcounting method in quantitative analysis of 2D image," in The Fractal Geometry of the Brain, A. Di Leva, Ed., pp. 109-127, Springer-Verlag, Berlin, Germany, 2016.

[8] V. Chan-Palay, Cerebellar Dentate Nucleus: Organization, Cytology and Transmitters, Springer, New York, NY, USA, 1977.

[9] N. T. Milošević, D. Ristanović, R. Gudović, K. Rajković, and D. Marić, "Application of fractal analysis to neuronal dendritic arborisation patterns of the monkey dentate nucleus," Neuroscience Letters, vol. 425, no. 1, pp. 23-27, 2007.

[10] B. Krstonošic, Morphological analysis of $2 D$ projections of neurons from the human neostriatum (Ph.D. thesis), Medical faculty, Univesity of Novi Sad, Serbia, Balkans, 2013.

[11] D. Maric, Qualitative and quantitative analysis of adult human dentate nucleus neurons morphology (Ph.D. thesis), Medical faculty, University of Novi Sad, Serbia, Balkans, 2010.

[12] T. G. Smith Jr., W. B. Marks, G. D. Lange, W. H. Sheriff Jr., and E. A. Neale, "A fractal analysis of cell images," Journal of Neuroscience Methods, vol. 27, no. 2, pp. 173-180, 1989.

[13] H. E. Taylor and T. L. Wade, University Calculus, John Wiley \& Sons, New York, NY, USA, 1962.

[14] H. F. Jelinek and E. Fernandez, "Neurons and fractals: how reliable and useful are calculations of offractal dimensions?" Journal of Neuroscience Methods, vol. 81, no. 1-2, pp. 9-18, 1998.

[15] K. Falconer, Fractal Geometry: Mathematical Foundation and Applications, John Wiley \& Sons, New York, NY, USA, 2nd edition, 2003.

[16] N. T. Milošević and D. Ristanović, "Fractality of dendritic arborization of spinal cord neurons," Neuroscience Letters, vol. 396, no. 3, pp. 172-176, 2006.

[17] H. F. Jelinek, G. N. Elston, and B. Zietsch, "Fractal analysis: pitfalls and revelations in neuroscience," in Fractals in Biology and Medicine IV, G. A. Losa, D. Merlini, T. F. Nonnenmacher, and E. R. Weibel, Eds., pp. 85-94, Birkhäuser, Basel, Switzerland, 2005.

[18] D. Ristanović, B. D. Stefanović, and N. Puškaš, "Fractal analysis of dendrite morphology of rotated neuronal pictures: the modified box counting method," Theoretical Biology Forum, vol. 107, pp. 109-121, 2014.

[19] N. Puškaš, I. Zaletel, B. D. Stefanović, and D. Ristanović, "Fractal dimension of apical dendritic arborization differs in the superficial and the deep pyramidal neurons of the rat cerebral neocortex," Neuroscience Letters, vol. 589, pp. 88-91, 2015. 
[20] D. Corforth and H. F. Jelinek, "Monofractal and multifractal analysis for interpretation of fuction-structure relationships in finite size biological material," in Classification and Application of Fractals, E. W. Mitchell and S. R. Murray, Eds., pp. 1-22, Nova Science Publishers, USA, 2011.

[21] T. Y. Zhang and C. Y. Suen, "A fast parallel algorithm for thinning digital patterns," Communications of the ACM, vol. 27, no. 3, pp. 236-239, 1984.

[22] R. H. Riffenburgh, Statistics in Medicine, Academic Press, London, UK, 1999.

[23] G. A. Losa, "The fractal geometry of life," Rivista Di Biologia, vol. 102, pp. 29-59, 2009.

[24] G. A. Losa, A. Di Ieva, F. Grizzi, and G. De Vico, "On the fractal nature of nervous cell system," Frontiers in Neuroanatomy, vol. 5, no. 1-2, 2011.

[25] T. G. Smith and G. D. Lange, "Fractal studies of neuronal and glial cellular morphology," in Fractal Geometry in Biological Systems: An Analytical Approach, P. M. Iannaccone and M. Khokha, Eds., pp. 173-186, CRC Press, New York, NY, USA, 1995.

[26] H. Nakayama, W. Kiatipattanasakul, S. Nakamura et al., "Fractal analysis of senile plaque observed in various animal species," Neuroscience Letters, vol. 297, no. 3, pp. 195-198, 2001.

[27] E. A. Neale, L. M. Bowers, and T. G. Smith, "Early dendrite development in spinal cord cell cultures: a Quantitative Study," Journal of Neuroscience Research, vol. 34, no. 1, pp. 54-66, 1993.

[28] M. Bizzarri, A. Giuliani, A. Cucina, F. D’Anselmi, A. M. Soto, and C. Sonnenschein, "Fractal analysis in a systems biology approach to cancer," Seminars in Cancer Biology, vol. 21, no. 3, pp. 175-182, 2011.

[29] J. B. Bassingthwaighte, L. S. Liebovitch, and B. J. West, Fractal Physiology, Oxford University Press, New York, NY, USA, 1994.

[30] A. Di Ieva, F. Grizzi, H. Jelinek, A. J. Pellionisz, and G. A. Losa, "Fractals in the neurosciences, part I: General principles and basic neurosciences," Neuroscientist, vol. 20, no. 4, pp. 403-417, 2014.

[31] H. F. Jelinek, N. T. Milošević, A. Karperien, and B. Krstonošić, "Box-counting and multifractal analysis in neuronal and glial classification," in Advances in Intelligent Control Systems and Computers Science, I. Dumitrache, Ed., Springer-Verlag, Berlin, Germany, p. 177, 2013. 


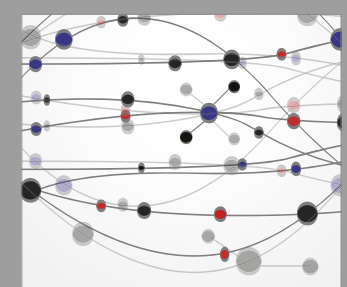

The Scientific World Journal
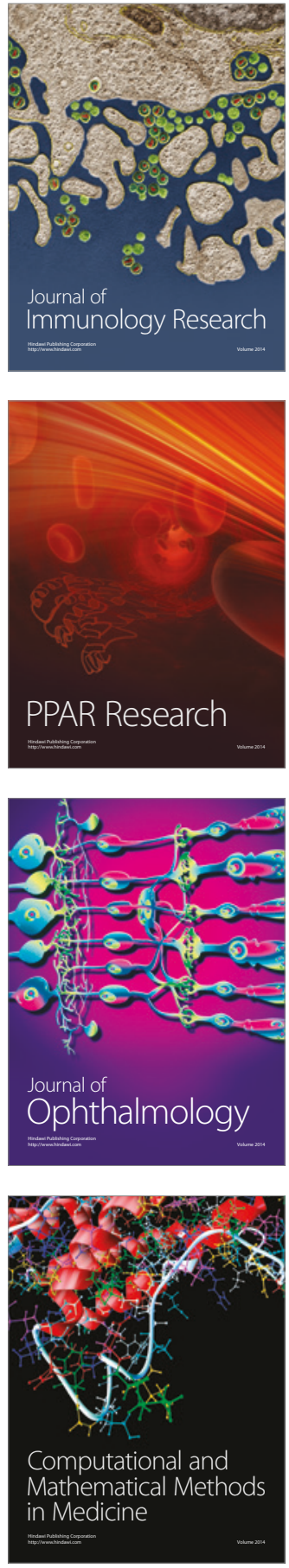

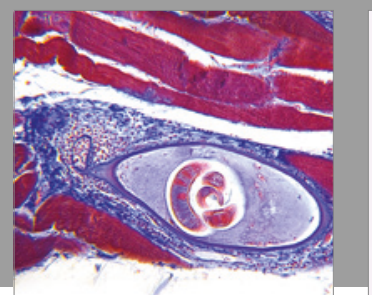

Gastroenterology Research and Practice
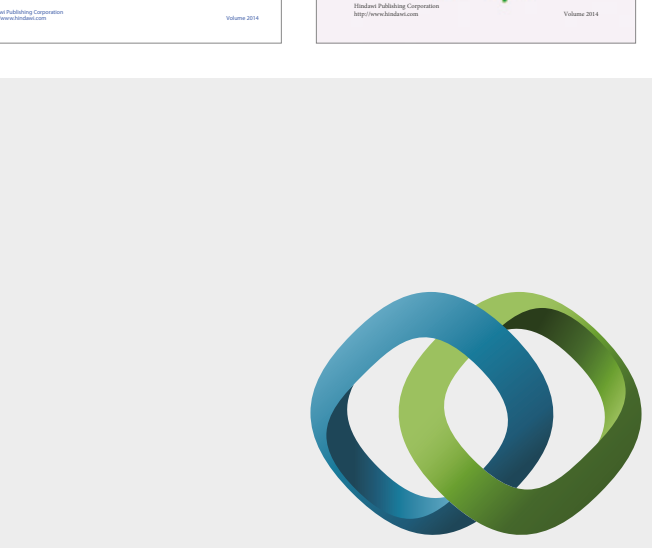

\section{Hindawi}

Submit your manuscripts at

https://www.hindawi.com
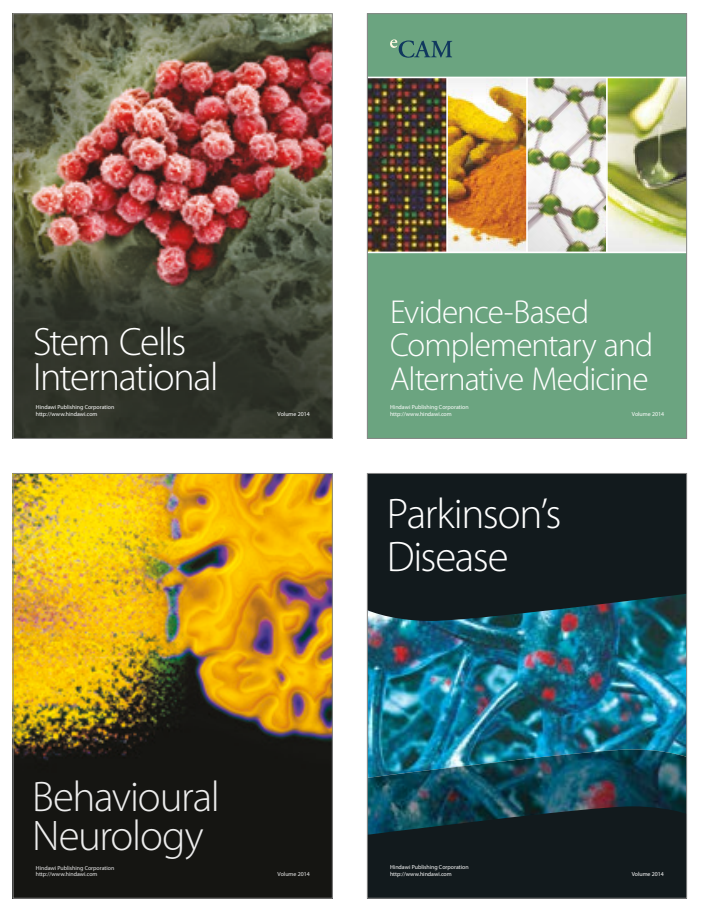
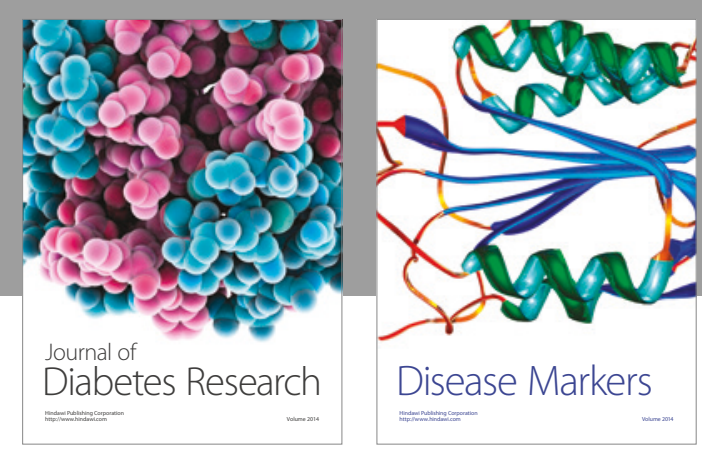

Disease Markers
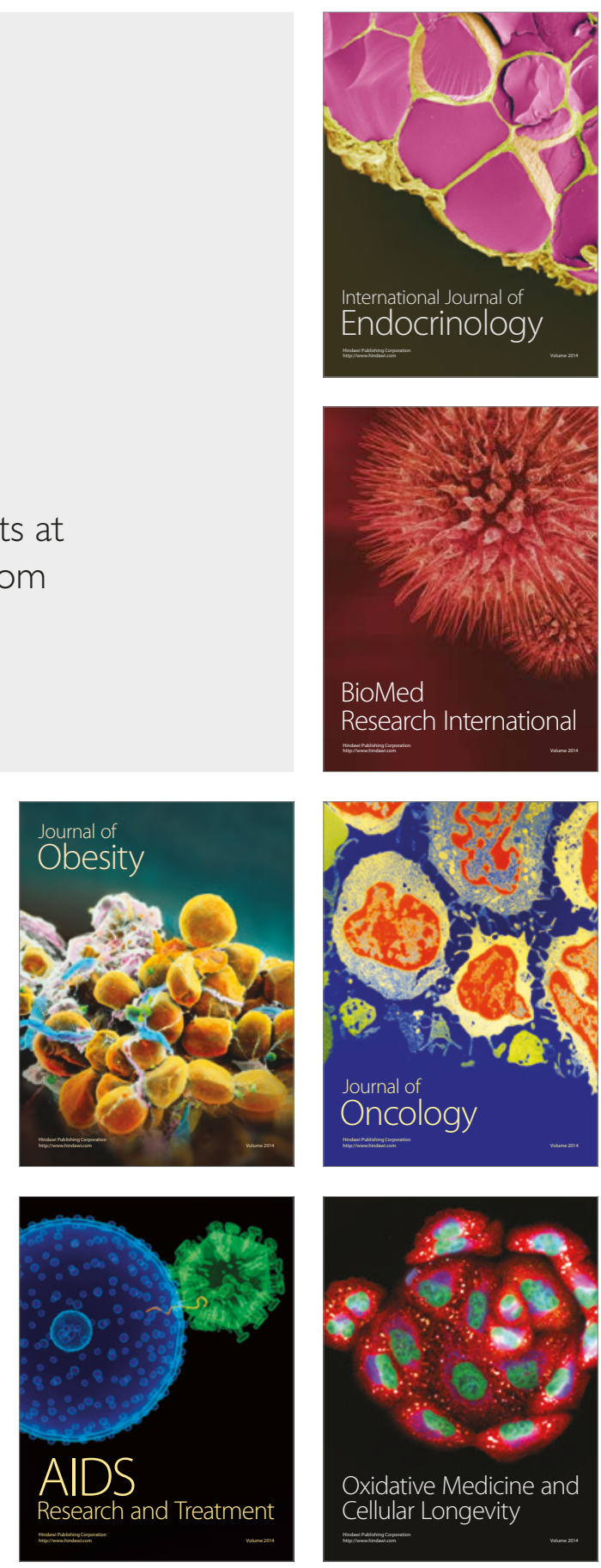